\title{
Botulinum toxin treatment for adductor spasmodic dysphonia with EMG and endoscopic guidance
}

\author{
${ }^{1}$ Thanh Tuan Nguyen, ${ }^{2}$ Ngoc Tai Tran, ${ }^{2}$ Truc Dung Nguyen, ${ }^{2}$ Thi Hung Nguyen \\ ${ }^{1}$ School of Medicine, Vietnam National University - Ho Chi Minh City; ${ }^{2}$ University Medical Center of \\ Ho Chi Minh City, Vietnam
}

\begin{abstract}
Background \& Objective: Spasmodic dysphonia is idiopathic focal dystonia characterized by irregular contractions or postural disorders of the laryngeal muscles, resulting in abnormal speech. Progress in laryngoscopy has resulted in development of a precise technique of botulinum toxin (BTX) injection into the thyroarytenoid muscle under visual control. Methods: We used EMG and endoscopic guidance for BTX injection in adductor spasmodic dysphonia (AdSD). Results: Thirty eight patients with AdSD were injected with BTX in 84 treatment sessions. The voice handicap index (VHI) level of the patients were: severe $(73.8 \%)$, moderate $(26.2 \%)$ and mild (none). The average VHI score was 74.6 points. After 8 weeks of BTX treatment, the VHI level was: Severe (3.6\%), moderate (10.8\%), and mild $(85.7 \%)$. The average VHI score was 27.7 points. The average values of jitter, shimmer and harmonic to noice ratio (HNR) improved statistically $(\mathrm{p}<0.05)$. The incidence of side effects were: breathiness (17.9\%), choking (15.5\%); hoarseness (8.5\%), and dysphagia (4.7\%).

Conclusions: Injection of BTX under both EMG and laryngoscopy guidance for treatment of adductor spasmodic dysphonia is an effective and safe treatment.
\end{abstract}

Keywords: Botulinum toxin, spasmodic dysphonia, electromyography, laryngoscopy

\section{INTRODUCTION}

Spasmodic dysphonia (SD) is an idiopathic focal dystonia characterized by irregular contractions or postural disorders of the laryngeal muscles, resulting in abnormal phonation. This is a rare disease, the estimated incidence is about $1 / 100,000$ population. ${ }^{1}$ SD has two forms: adductor spasmodic dysphonia (AdSD) and abductor SD. AdSD is more common, with closing the glottis, resulting in strangled, stiffened and interruption of voice. ${ }^{2}$

SD is diagnosed clinically with no gold diagnostic standard. In the past, oral medications were used for the treatment of SD but they were not effective. Since 2016, the American Academy of Otolaryngology-Head and Neck Surgery has recommended using Botulinum toxin A (BTX-A) for treatment $\mathrm{SD}$. It is currently regarded as the first choice therapy. ${ }^{3}$

Dysport ${ }^{\circledR}$ is a BTX-A that has been studied in many countries around the world through various methods of investigations. Most results show that BTX-Ais safe and effective in SD patients. ${ }^{4,5}$
In Vietnam, in recent years, several hospitals have started treating patients with dystonia with BTX-ABTX. BTX is commonly delivered by percutaneous injection under EMG guidance. Recently, progress in laryngoscopy has resulted in the development of a precise technique where BTX is injected directly into the thyroarytenoid muscle under visual control. It remains to be determined which route is better. The traditional technique under EMG guidance is simple, effective, and safe. This is the report of treatment using BTX with additional laryngoscopy guidance

\section{METHODS}

This retrospective review was performed for patients with SD treated with BTX in the laryngology clinic at the HCMC University Medical Center and HCMC Ear Nose Throat Hospital from June 2018 to June 2020. Patients selected for analysis were those that had undergone treatment for AdSD by Dysport $\AA$.

The inclusion criteria were: All patients from 18 years old diagnosed with SD by physicians 
who were experienced with SD. The patient has not been treated with BTX previously, or the minimum time since the last BTX was more than 12 weeks, or the patient's condition has recurred. The patient treatment with BTX as decided by the physician, and there was no intervention by the investigators.

The exclusion criteria were: Patients with severe medical conditions or those taking the aminoglycoside antibiotics; who experienced difficulty swallowing before treatment for any cause ; patients with neuromuscular disease or other associated neurological disorders (for example: myasthenia gravis, Eaton-Lambert syndrome, motor neuron disease affects neuromuscular nodes).

\section{BTX preparation and administration}

The BTX-A administered was with the brand name Dysport ${ }^{\circledR}$, manufactured by Ipsen Lt.. BTX injection was given bilaterally, same dose for each thyroarytenoid muscle. BTX-A was injected under both EMG and endoscopic guidance. An endoscopist provided flexible laryngoscopy for injection. The patients were placed in a sitting position with their neck slightly extended.A $37 \mathrm{~mm}$ 26-gauge monopolar, hollow-bore, Teflon-coated EMG needle was injected percutaneously through the cricothyroid membrane and into the area of the thyroarytenoid muscle. (Figure 1)

\section{RESULTS}

From June 2018 to June 2020, patients treated with BTX type A for a diagnosis of AdSD were included in this study. Those without adequate documentation of dose effect was excluded. Thirty eight subjects who had a total of 84 BTX injections for AdSD were included in this study. All injections were performed bilaterally into the adductor thyroarytenoid muscle under both EMG and endoscopic guidance.

Demographics of the study patients are shown
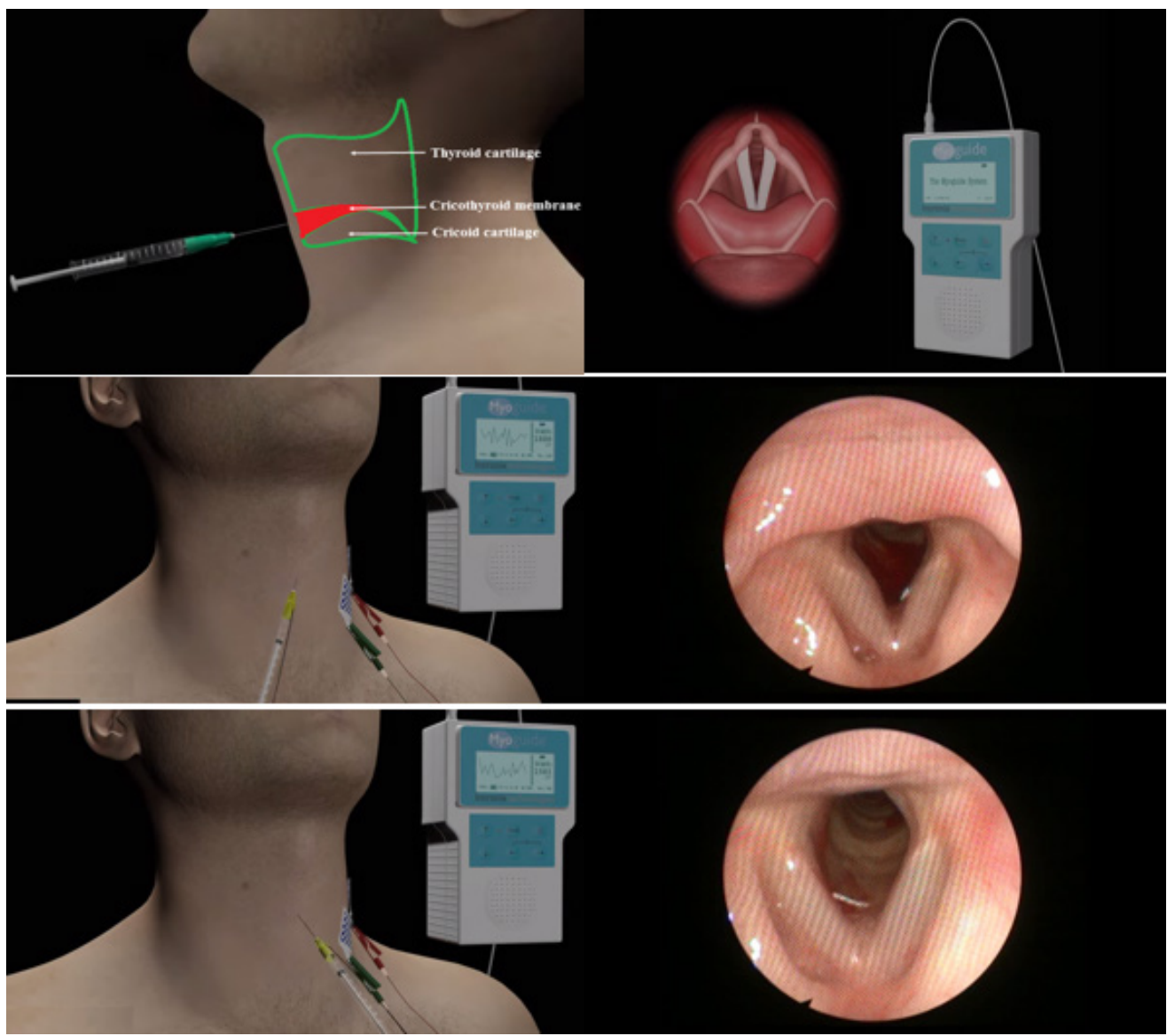

Figure 1. Technique of bilateral BTX-A injection for treatment of adductor spasmodic dysponia under both EMG and endoscopic guidance 
Table 1: Characteristic of patients with adductor spasmodic dysphonia

\begin{tabular}{lc}
\hline Characteristics & $\mathbf{n = 3 8}$ \\
\hline Age in years & $54.5 \pm 10.8$ \\
Female/male & $22 / 2$ \\
BMI & $20.8 \pm 2.2$ \\
Duration of illness (years) & $2.9 \pm 3.7$ \\
Family history of movement disorder & $1(4.2 \%)$ \\
\hline
\end{tabular}

in Table 1 . There were $35 / 38$ patients $(92.1 \%)$ with persistent dysphonia and 3/38 (8\%) intermittent dysphonia. Time from onset of symptom to treatment was $2.9 \pm 3.7$ years (shortest was 3 months, longest was 15 year). The interval was more than 5 years $(23.7 \%), 2$ to 5 years $(28.9 \%)$, 1 to 2 years $(31.6 \%)$, less than 1 year $(15.8 \%)$.

Results of BTX injections are shown in Table 2. Regarding the degree of SD according to voice handicap index (VHI), it consisted of: severe (73.8\%), moderate (26.2\%), mild (none). As for the dysphonia's functional effects, it resulted in aphonia (100\%), communication effects $(92.1 \%)$, and reduced income $(81.6 \%)$. As for prior treatment, $78.9 \%$ of patients received speech therapy; $81.6 \%$ received oral drug treatment before the BTX injection. Regarding acoustic analysis before BTX injection, the average value of jitter was 2.6; shimmer was 15.8 and harmonic to noice ratio (HNR) was 14.1.

The average number of injections was 2.2 times, the maximum was 6 times. The mean effective duration was 4.3 months,; the shortest was 1 month and the longest was 8 months.

Of the 84 injections, there were no major complications such as requiring endotracheal intubation, tracheostomy, or hospitalization related to the airway. The side effects were: breathiness
(17.9\%); aspiration (15.5\%); hoarseness $(9.5 \%)$; dysphagia (4.7\%) and pain (3.6\%). Typically, the toxin's effect occurs within the first 48-72 hours.

Before BTX injection, the average VHI score was 74.6. After injection, the average VHI score was 27.7 (average decrease of 46.9 points). After 8 weeks, the VHI score was severe (3.6\%), moderate $(10.7 \%)$, slight $(85.7 \%)$. The improvement in VHI after BTX-A injection was significant $(\mathrm{p}<0.05)$.

In terms of acoustic analysis, after treatment, all 3 indicators improved significantly: jitter decreased to 1.2 , shimmer decreased to 9.4 and HNR increased to 17.9 ( $p<0.05$ ). Regarding patient post-treatment satisfaction, $71.4 \%$ of patients were highly satisfied, $21.4 \%$ moderate, and $7.1 \%$ were not satisfied.

As an overall assessment, the rate of good improvement was $67.9 \%$; moderate improvement was $25.0 \%$ and no improvement was $7.1 \%$.

\section{DISCUSSION}

The average age of our patients was 54 years, the oldest was 73 years, the youngest was 22 years. The age group from 41-60 years old accounted for $42.0 \%$. Similar results has been reported from elsewhere such as Emilyeh et al. ${ }^{4}$ with an average age of 54.4 years $( \pm 16.7)$.

Table 2: Treatment of adductor spasmodic dysphonia with BTX injections

\begin{tabular}{lc}
\hline Number of AdSD patients & $\mathbf{n}=\mathbf{3 8}$ \\
\hline Technique & EMG and endoscopic guidance \\
Total number of injections & 84 \\
Average number of injections & $2.2 \pm 1.7(1-6)$ \\
1 injection & $45.2 \%$ \\
2 injection & $26.2 \%$ \\
3 injection & $14.3 \%$ \\
4 injection & $7.1 \%$ \\
6 injection & $2.4 \%$ \\
Average dose per side for bilateral injections & 2.5 Units (Dysport) \\
\hline
\end{tabular}


As for gender, our study consisted of 36 female and 2 male. The preponderance of SD among females has been noted previously. Elmiyeh et $a l .{ }^{4}$ reported a female preponderance of $62 \%$ and Zwirner et al. ${ }^{6}$ reported a female preponderance of $90.9 \%$. This may be partly explained by the triggering factors at the onset; i.e., psychosocial stress and pregnancy.

In this study, all 38 patients were of the AdSD, there was no case of abductor SD. Abductor SD is known to be less common. In Abductor SD, spasms cause the vocal folds to remain open. The vocal folds cannot vibrate when they are open too far. The open position also allows air to escape from the lungs during the speech. As a result, the voice became weak and breathy. As with adductor spasmodic dysphonia, the spasms are often absent during activities such as laughing, crying, or whispering. According to Tanner et al. on 150 patients, AdSD accounted for $98.7 \%$, with only 2 patients $(1.3 \%)$ of AbSD. ${ }^{7}$ Similarly, Tisch et al. reported only studied 169 patients with SD, only $3 / 169$ (1.8\%) patients with Abductor SD, the restwere AdSD (98.2\%). ${ }^{8}$

As for onset of symptom to injection, the duration of less than 1 year accounted for only $15.8 \%$. This could be due to lack of awareness of BTX as effective treatment, and a delay in the diagnosis of SD. ${ }^{9}$

Injection of BTX into vocal folds is considered the first-choice treatment for AdSD. ${ }^{10}$ According to previous reports, this treatment produces an improvement of voice quality in $80 \%-100 \%$ of patients. ${ }^{3,11}$ To be effective on AdSD, BTX must be injected in the vocal folds and precisely into the thyroarytenoid muscle ${ }^{12}$, where it must induce chemo denervation. The most common technique is trans cricoid access by EMG guidance ${ }^{13}$, which allows monitoring of muscle activity during phonation. Individual anatomical differences of the muscle or possible concurrent dystonia of extra phonatory muscles may lead to mislocalization of the injection site with consequent varying injection efficacy, as reported by some studies.

Two types of BTX type A: Botox ${ }^{\circledR}$ (Allergan) or Dysport ${ }^{\circledR}$ (Ipsen) have been used for AdSD. In this report, BTX-A Dysport ${ }^{\circledR}$ (Ipsen) injection was used with both EMG and endoscopic guidance. Theoretically, technique under single EMG guidance may misidentify the injection site. Ruiz et al. reported the effectiveness of the treatment of BTX by transcutaneous injection in 61 of 76 injections, but 48/48 injections with direct vision by transoral route. Changes in vocal cord position during injection or anatomical abnormalities are the cause of failure in transcutaneous injection. ${ }^{14}$
In the AdSD, two vocal cords become constricted and tend to close the glottis that causes the needle to be inserted into the wrong position, usually into the cricothyroid space at the midline position. During the injection, patients are often tense, they keep the vocal cords open - under force in breathing. So that the authors often recommend placing the needle at an angle of $15^{\circ}-30^{\circ}$ with the sagittal plane. This helps the injection site into the correct vocal cords or the cavity around the larynx muscle, or at least into the lateral cricoarytenoid muscle. Denervation of this muscle reduces the inward pull force that closes the vocal cords, without causing dystonia in the thyroarytenoid muscle. Failure of BTX treatment may also be due to reflexes of the pharynx and larynx muscles triggered by needle tip movements and may leak BTX into the larynx. ${ }^{15}$

The technique of BTX injection injected into the muscular thyroid muscle under the laryngoscopy image guide is almost of no risk, ${ }^{14}$ because the injection process always observes the bilateral vocal cords and determines the exact location to be injected. Then inject BTX into the muscular thyroid just below the mucosa; this is confirmed by an image of a submucosal bulge or bleeding at the site of a needle prick. This technique also helps to bring BTX directly into the vocal muscle (the innermost part of the thyroarytenoid muscle). Laryngoscopy guidance may also be valuable as a teaching tool. A teaching clinician can easily assess the accuracy of needle placement with a remote EMG monitor and video laryngoscopy.

This injection technique is based on two assumptions: 1) This technique allows BTX to be precisely injected visually so that the ability to diffuse the field of motor endplates in the thyroarytenoid muscle is maximized with minimal doses. 2) Patients may prefer this technique since it is well tolerated by most and, if the dosage is reduced, there is less likelihood of dose-related side effects such as breathiness. However, the disadvantage of this technique is greater complexity. It requires the training or coordination of the endoscopist. ${ }^{16}$ Thus, the choice of injection technique should be based on availability, convenience, and the staff of the healthcare center.

In conclusion, despite its greater complexity, the technique under both EMG and laryngoscopy guidance is probably more effective and safer than the technique under single EMG guidance. We describe the result of this technique from a centre in Vietnam, that is composed of simple elements available at many medical center. 


\section{REFERENCES}

1. Schweinfurth JM, Billante M, Courey MS. Risk factors and demographics in patients with spasmodic dysphonia. Laryngoscope 2002;112(2):220-3.

2. Ludlow CL. Spasmodic dysphonia: a laryngeal control disorder specific to speech. J Neurosci 2011;31(3):793-7.

3. Whurr R, Lorch M. Review of differential diagnosis and management of spasmodic dysphonia. Curr Opin Otolaryngol Head Neck Surg 2016;24(3):203-7.

4. Elmiyeh B, Prasad VM, Upile T, et al. A singlecentre retrospective review of unilateral and bilateral Dysport ${ }^{\circledR}$ injections in adductor spasmodic dysphonia. Logoped Phoniatr Vocol 2010;35(1):3944.

5. Upile T, Elmiyeh B, Jerjes W, et al. Unilateral versus bilateral thyroarytenoid Botulinum toxin injections in adductor spasmodic dysphonia: a prospective study. Head Face Med 2009;5(1):20.

6. Zwirner P, Murry T, Swenson M, Woodson GE. Effects of botulinum toxin therapy in patients with adductor spasmodic dysphonia: acoustic, aerodynamic, and videoendoscopic findings. Laryngoscope 1992;102(4):400-6.

7. Tanner K, Roy N, Merrill RM, Sauder C, Houtz DR, Smith ME. Spasmodic dysphonia: onset, course, socioemotional effects, and treatment response. Ann Otol Rhinol Laryngol 2011;120(7):465-73.

8. Tisch S, Brake H, Law M, Cole I, Darveniza P. Spasmodic dysphonia: clinical features and effects of botulinum toxin therapy in 169 patients - an Australian experience. J Clin Neurosci 2003;10(4):434-8.

9. Creighton FX, Hapner E, Klein A, Rosen A, Jinnah HA, Johns MM. Diagnostic delays in spasmodic dysphonia: a call for clinician education. $J$ Voice 2015;29(5):592-4.

10. Blitzer A, Brin MF, Stewart CF. Botulinum toxin management of spasmodic dysphonia (laryngeal dystonia): a 12-year experience in more than 900 patients. Laryngoscope 1998;108(10):1435-41.

11. Aronson AE, Mccaffrey TV, Litchy WJ, Lipton RJ. Botulinum toxin injection for adductor spastic dysphonia: patient self-ratings of voice and phonatory effort after three successive injections. Laryngoscope 1993;103(6):683-92.

12. George EF, Zimbler M, Biller HF, Wu BL, Sanders I. Quantitative mapping of the effect of botulinum toxin injections in the thyroarytenoid muscle. Ann Otol Rhinol Laryngol 1992;101(11):888-92.

13. Blitzer A, Briny MF, Fahn S, Lovelace RE. Clinical and laboratory characteristics of focal laryngeal dystonia: study of 110 cases. Laryngoscope 1988;98(6):636-40.

14. García PR, Cenjor CE, Sanchez VB, Astarloa R, Sanabria J, de Yébenes García J. Botulinum toxin treatment for spasmodic dysphonia: percutaneous versus transoral approach. Clin Neuropharmacol 1998;21(3):196-8.

15. Galardi G, Guerriero R, Amadio S, et al. Sporadic failure of botulinum toxin treatment in usually responsive patients with adductor spasmodic dysphonia. Neurol Sci 2001;22(4):303-6.
16. Ford CN, Bless DM, Lowery JD. Indirect laryngoscopic approach for injection of botulinum toxin in spasmodic dysphonia. Otol Head Neck Surg 1990;103(5):752-8. 